\title{
Real-time all-optical ultrasound imaging of a dynamic heart valve phantom
}

Colchester, Richard, Moore, John, Peters, Terry, Finlay, Malcolm, Desjardins, Adrien

Richard J. Colchester, John T. Moore, Terry M. Peters, Malcolm C. Finlay, Adrien E. Desjardins, "Real-time all-optical ultrasound imaging of a dynamic heart valve phantom," Proc. SPIE 11879, Frontiers in Biophotonics and Imaging, 1187908 (6 October 2021); doi: 10.1117/12.2598781

SPIE. Event: SPIE Photonex, 2021, Glasgow, Scotland, United Kingdom 


\title{
Real-time all-optical ultrasound imaging of a dynamic heart valve phantom
}

\author{
Richard J. Colchester ${ }^{\mathrm{a}, \mathrm{b}}$, John T. Moore ${ }^{\mathrm{c}}$, Terry M. Peters ${ }^{\mathrm{c}}$, Malcolm C. Finlay ${ }^{\mathrm{b}, \mathrm{d}}$, and Adrien \\ E. Desjardins ${ }^{\mathrm{a}, \mathrm{b}}$ \\ aDepartment of Medical Physics and Biomedical Engineering, University College London, \\ London, UK \\ ${ }^{\mathrm{b}}$ Wellcome/EPSRC Centre for Interventional and Surgical Sciences, University College \\ London, London, UK \\ ${ }^{c}$ Robarts Research Institute, Western University, London Ontario, Canada \\ ${ }^{\mathrm{d}}$ William Harvey Cardiovascular Research Institute, Queen Mary University of London and \\ Barts Health Centre London, London, United Kingdom
}

\begin{abstract}
All-optical ultrasound imaging, in which ultrasound is generated and received using light, is well-suited to minimally invasive surgical procedures. Here we present a device that can provide real-time M-mode ultrasound images, and demonstrate its use imaging a dynamic heart valve phantom. This device, comprising two optical fibres, one with a graphene-polydimethylsiloxane composite coating for ultrasound generation, and a second with a concave Fabry-Pérot cavity for ultrasound reception, had a diameter of $<1 \mathrm{~mm}$. This provided a wide ultrasound transmission bandwidth $(>30 \mathrm{MHz})$ that enabled imaging with high axial resolution $(<50 \mu \mathrm{m})$ and large imaging depths $(>2 \mathrm{~cm})$. M-mode imaging with an A-line rate of $100 \mathrm{~Hz}$ was demonstrated on a heart valve phantom with realistic mitral valve motion. This work demonstrates the potential for all-optical ultrasound imaging to be used for guidance of intracardiac interventions.
\end{abstract}

Keywords: Ultrasound imaging, All-optical ultrasound, Heart phantom, Minimally invasive imaging

\section{INTRODUCTION}

New methods for imaging from inside the body can be transformative for minimally invasive surgery. A prominent example is endovascular cardiac valve surgery, where identifying tissue structures in relation to medical devices used for minimally invasive surgery can be critical to ensure positive outcomes. Ultrasound imaging has been used in this context, and M-mode imaging has been demonstrated for diagnosis of ventricular disorders. ${ }^{1}$ One emerging modality which is well-suited to application for these procedures is all-optical ultrasound (OpUS). While traditional intracardiac and transoesophageal echocardiography (ICE and TOE) provide standard of care valve imaging, OpUS offers the ability to use probes and catheters with a lower profile, making it possible to access much smaller vessels (e.g., the coronary sinus), and reducing the overall invasiveness of ICE and even TOE imaging. With OpUS, ultrasound is both generated and received using light. For ultrasound generation, pulsed or modulated laser light is absorbed within a medium which leads to a temperature rise and a corresponding pressure rise due to the photoacoustic effect. ${ }^{2,3}$ This pressure then propagates as ultrasound. For ultrasound reception, resonant optical cavities can be used. ${ }^{4}$ With these devices, the impinging ultrasound causes small deformations to the cavity which can be monitored with a high sensitivity. Unlike its electronic counterpart, OpUS can be readily miniaturised through the use of optical fibres without compromising the device performance. Broadband ultrasound and a high sensitivity can be maintained whilst achieving sub-millimetre lateral device dimensions. ${ }^{3,5-10}$

Recent work has demonstrated the efficacy of this technology for ex vivo imaging, both in $2 \mathrm{D}$ and $3 \mathrm{D} .{ }^{3-5}$ Further, rotational 2D images were acquired by Colchester et al. in a ex vivo swine carotid artery. ${ }^{6}$ However, in

Further author information: (Send correspondence to R.J.C.)

R.J.C.: E-mail: richard.colchester@ucl.ac.uk

Frontiers in Biophotonics and Imaging, edited by Sumeet Mahajan, Stefanie Reichelt, Proc. of SPIE

Vol. 11879, 1187908 - (c) 2021 SPIE · CCC code: 0277-786X/21/\$21 - doi: 10.1117/12.2598781 
that study, the acquisition rate was limited to $<10$ frames per second, which is not sufficient for cardiac valve imaging. An alternative method, demonstrated by Finlay et al. with an in vivo swine model, is M-mode imaging. ${ }^{8}$ Here, acquired ultrasound A-lines are concatenated in time to form a scrolling image. That work demonstrated the ability to image complex structures within an animal model. In this study, we present a similar device, which uses a highly directional OpUS transmitter to provide real-time M-mode imaging. A dynamic heart valve phantom was imaged to demonstrate the efficacy of this device for tracking valve movements.

\section{EXPERIMENTAL}

\subsection{All-Optical Ultrasound Probe}

An all-optical ultrasound probe was fabricated. The probe comprised a fibre optic ultrasound transmitter ${ }^{11}$ and a concave Fabry-Pérot fibre optic hydrophone. ${ }^{4}$ The fibre optic ultrasound transmitter comprised a 600 um core diameter optical fibre (FP600URT, Thorlabs, UK), with a composite coating of reduced graphene oxide (rGO) and polydimethylsiloxane (PDMS). ${ }^{11}$ The ultrasound transmitter generated ultrasound pressures in excess of $4 \mathrm{MPa}$ peak-to-peak at $1.5 \mathrm{~mm}$ from the coating surface, with a corresponding $-6 \mathrm{~dB}$ ultrasound bandwidth $>20 \mathrm{MHz}$. The transmitter was chosen due to its high directionality, previously reported by Colchester et al. ${ }^{11}$ The Fabry-Pérot fibre optic hydrophone comprised a single mode optical fibre (SMF-28) coated with a cavity which consisted of a planar dielectric mirror on the fibre end face, overcoated with a polymer (NOA81, Norland Optics, USA) dome, and subsequently a second dielectric mirror. ${ }^{4}$ These receivers have high sensitivity (NEP $<100 \mathrm{~Pa}$ ), broad receiver bandwidth $(>20 \mathrm{MHz})$, and exhibit an omnidirectional frequency response. ${ }^{4}$

To construct the imaging probe, the transmitter and receiver were aligned at their distal ends and held together with several $1 \mathrm{~cm}$ sections of heat shrink tubing spaced along the device body. This gave the device a maximum lateral diameter of $<1 \mathrm{~mm}$. The device size was dictated by the trade-off between the small lateral dimensions needed to navigate to the heart in a clinical context and the directionality of the transmitted ultrasound achieved using large ultrasound generation apertures. ${ }^{11}$ For protection and manipulation during the experiments, the fibres were housed in a metal hypotube (length: $30 \mathrm{~cm}$, inner diameter: $2.4 \mathrm{~mm}$, outer diameter: $2.75 \mathrm{~mm}$ ) with a y-piece luer connector on the proximal end to allow flushing (Figure 1). The hypotube was chosen due to its availability and compatibility with the heart phantom used in the study.

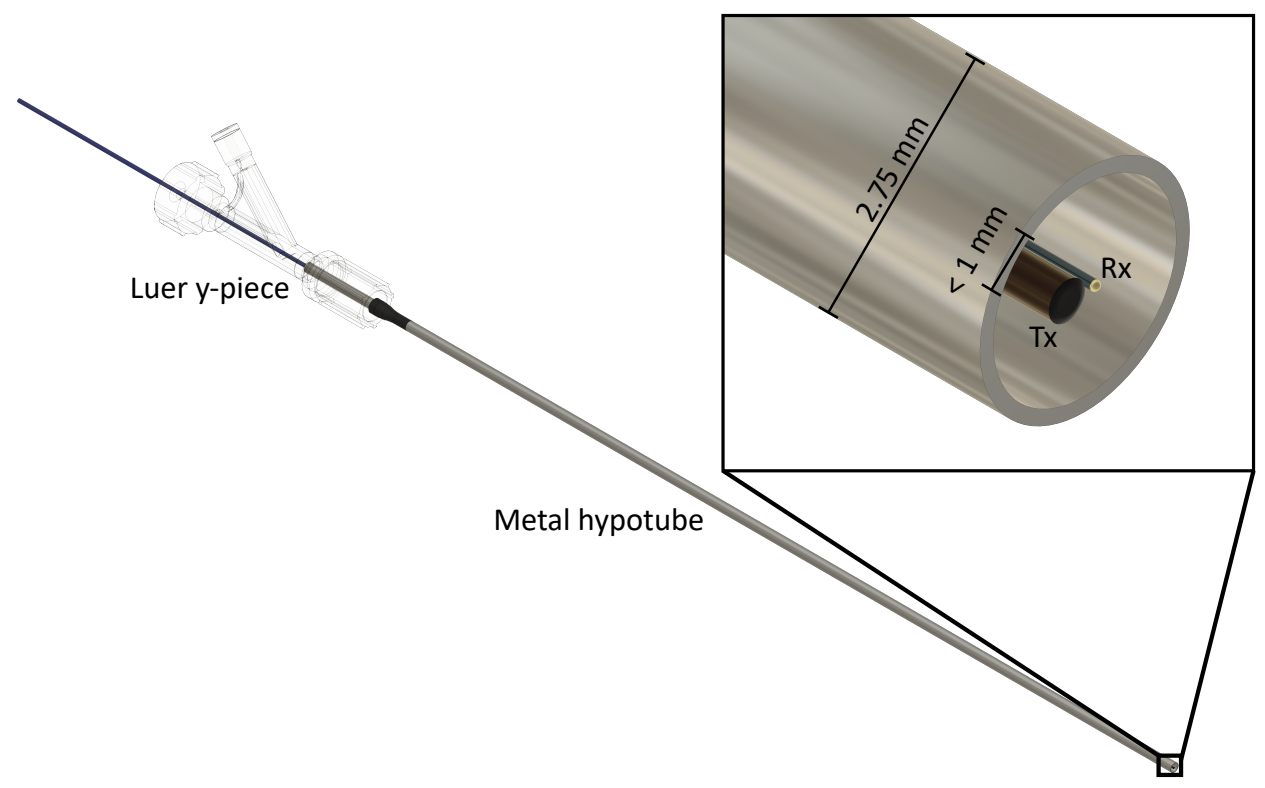

Figure 1. Schematic render of the all-optical ultrasound probe with metal hypotube housing and luer y-piece connector to enable flushing. Inset: close-up of the all-optical ultrasound transducer, with the reduced graphene oxide/polydimethylsiloxane transmitter $(\mathrm{Tx})$ and concave Fabry-Pérot hydrophone $(\mathrm{Rx})$. 


\subsection{Imaging System}

The device was connected to an all-optical ultrasound console which comprised hardware for ultrasound transmission and reception, and a PC for hardware control, data processing, and image display. Ultrasound was generated using a pulsed laser for photoacoustic excitation (wavelength: $1064 \mathrm{~nm}$, pulse length: $1.6 \mathrm{~ns}$, pulse energy: $40 \mu \mathrm{m}$, DSS-1064 Q, Crylas, Germany). Ultrasound reception was performed using a continuous wave tuneable laser (wavelength range: 1520-1560 nm, power: $20 \mathrm{~mW}$, TSL-550, Santec, UK) which was connected to the hydrophone via a circulator. Reflected optical signals from the hydrophone where measured using a photodiode. The low-frequency component of the signal was used to track the optimum bias point of the sensor. The high-frequency component of the signal was digitised at 14 bits and $125 \mathrm{MS} / \mathrm{s}$ using a high-speed digitiser (M4i.4420-x8, Spectrum, Germany).

The acquired ultrasound A-lines were frequency filtered (band pass $3-30 \mathrm{MHz}, 4^{\text {th }}$ Order, Butterworth) and crosstalk was removed using a previously described algorithm. ${ }^{5}$ Subsequently, signal suppression was applied to the first 400 samples to remove residual crosstalk. ${ }^{8}$ Then the data was Hilbert transformed to obtain the signal envelope and log transformed for display. For real-time display, the A-lines were concatenated in time and displayed as an M-mode image with an A-line rate of $100 \mathrm{~Hz}$. The raw data was also saved for offline processing.

\subsection{Heart valve phantom imaging}

A heart valve phantom (LV+ Simulator) developed by Archetype BioMedical (London, Ontario, Canada) was used (Figure 2 a)). ${ }^{12}$ The phantom comprised a silicon heart chamber which was actuated using six hydraulic pistons. Actuation of the heart chamber caused the opening and closing of synthetic mitral and aortic valves (Figure $2 \mathrm{~b}$ )). The valve leaflets could be adjusted by varying the tension on cords attached to them. For the experiments carried out here, the valve leaflets where adjusted to represent a healthy patient and the heart rate was set to 70 beats per minutes.

For the imaging experiments, the OpUS device was inserted in to the phantom through a silicone side port (Figure 2 a)). The device was flushed with water through the y-piece luer connector. The device tip was aligned with the valve such that the longitudinal axis of the device was approximately orthogonal to the valve surface when the valve was shut (Figure $2 \mathrm{~b}$ )), mimicking a surgical scenario. During image acquisition, the OpUS device was held stationary using a clamp. The mitral valve (MV) was imaged in 3 locations (Figure $2 \mathrm{c}$ )): anteriorly (MVA), centrally (MVC) and posteriorly (MVP); the aortic valve (AV) was imaged in a single location (Figure $2 \mathrm{c})$ ), on the non-coronary cuspid (NCC). All images were displayed in real-time and saved for offline processing. For each position $10 \mathrm{~s}$ of M-mode image data was acquired, corresponding to $1000 \mathrm{~A}$-lines.

\section{RESULTS}

All-optical ultrasound videos where acquired at four locations on the heart valve phantom (Figure 2 c)). A snapshot of each of the resulting videos is shown in Figure 3. At each of the locations, the valve leaflet being imaged was visible throughout the cardiac cycle and the full thickness of the valve was resolved. The valve motion could be tracked in real-time and crucially the structures were still visible at the extreme of the valve motion, when the angle between the device and the valve was oblique.

As visualised by OpUS (Figure 3 a)), the valve motion was seen to have the greatest range $(>1 \mathrm{~cm})$ at the central location on the mitral valve (MVC). When closed, i.e. closest to the OpUS device, the valve appeared as a thick $(c a .5 \mathrm{~mm})$ region on the ultrasound image. Whilst at the furthest extent, when fully open, the valve wall was only just visible, due to the oblique angle between the valve and the device. For both the anterior and posterior positions on the mitral valve (MVA \& MVP) (Figure $3 \mathrm{~b}$ ),c)) the valve wall was closer to the device for the majority of the cardiac cycle, as imaged by OpUS. Similar to the MVC image, the valve remained visible throughout the entire cardiac cycle, even at a depth of $15 \mathrm{~mm}$ (Figure $3 \mathrm{~b}$ )). When imaging the NCC on the aortic valve, the full valve motion could be visualised (Figure $3 \mathrm{~d}$ )). The NCC motion was different to that of the mitral valve and the subsurface signal presented as a lower intensity signal. 
a)
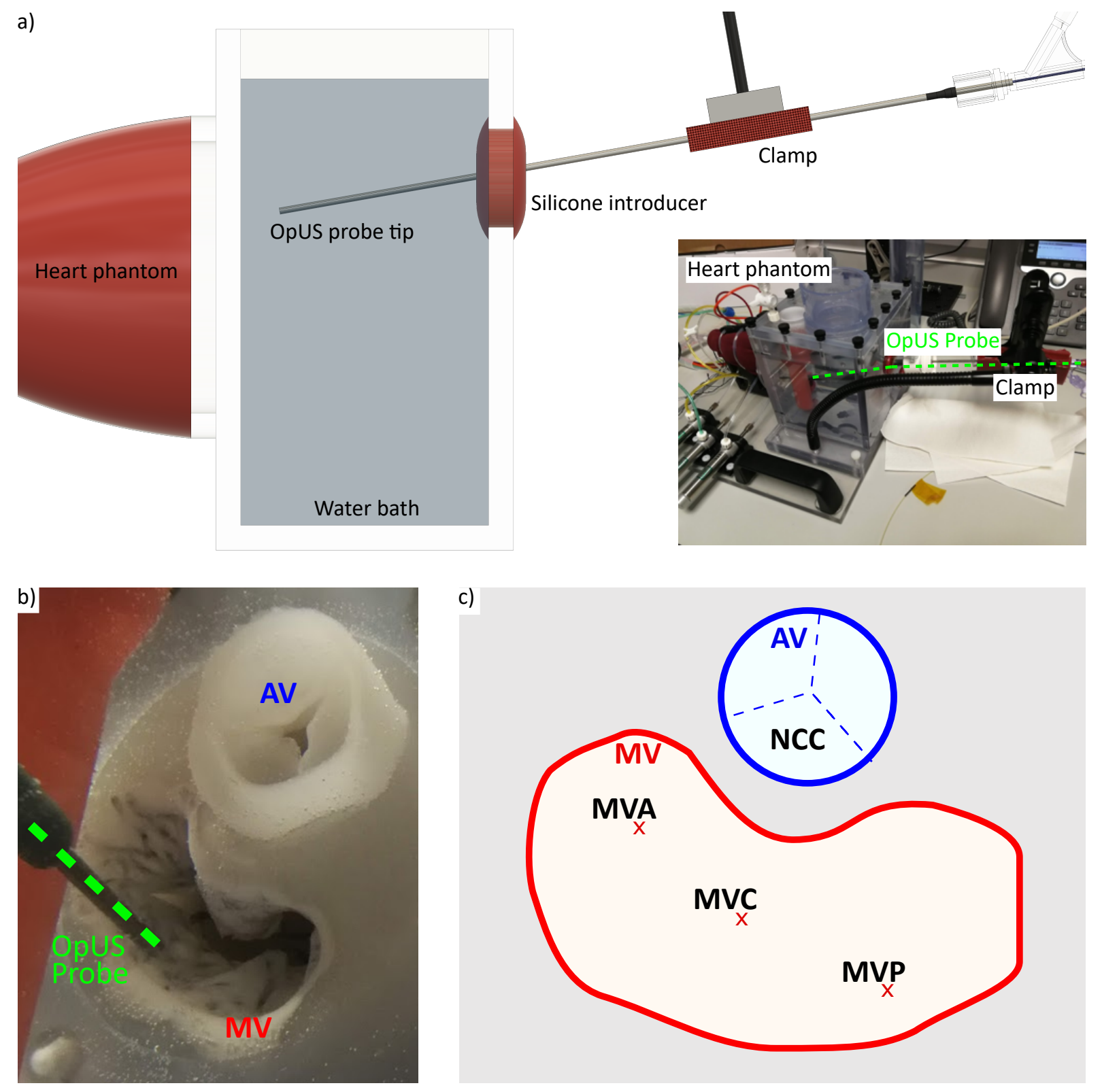

Figure 2. a) Side view schematic of the heart valve phantom, showing the all-optical ultrasound device inserted through a side port and clamped in place. Inset: photograph of the setup in the lab. b) Close-up photograph of the all-optical ultrasound device tip directed at the mitral valve (MV), with the aortic valve above (AV). c) Schematic map of the locations on the mitral and aortic valves that were imaged using the all-optical ultrasound device.

\section{DISCUSSION AND CONCLUSION}

Here we presented the first M-mode OpUS imaging study of heart valve dynamics. For this study, an OpUS imaging device for real-time M-mode imaging at $100 \mathrm{~Hz}$ was fabricated. The device comprised a directional rGO-PDMS ultrasound transmitter coupled with a Fabry-Pérot hydrophone. The device was housed in metal hypotube for protection and to facilitate imaging of the phantom. To demonstrate real-time imaging, a dynamic heart valve phantom was used. The phantom comprised a heart chamber and mitral and aortic valves. The heart chamber was actuated to provide valve movement which mimicking a healthy patient. Here a heart rate of $70 \mathrm{BPM}$ was chosen, but this could be modified in future experiments. Further, different anatomical and 

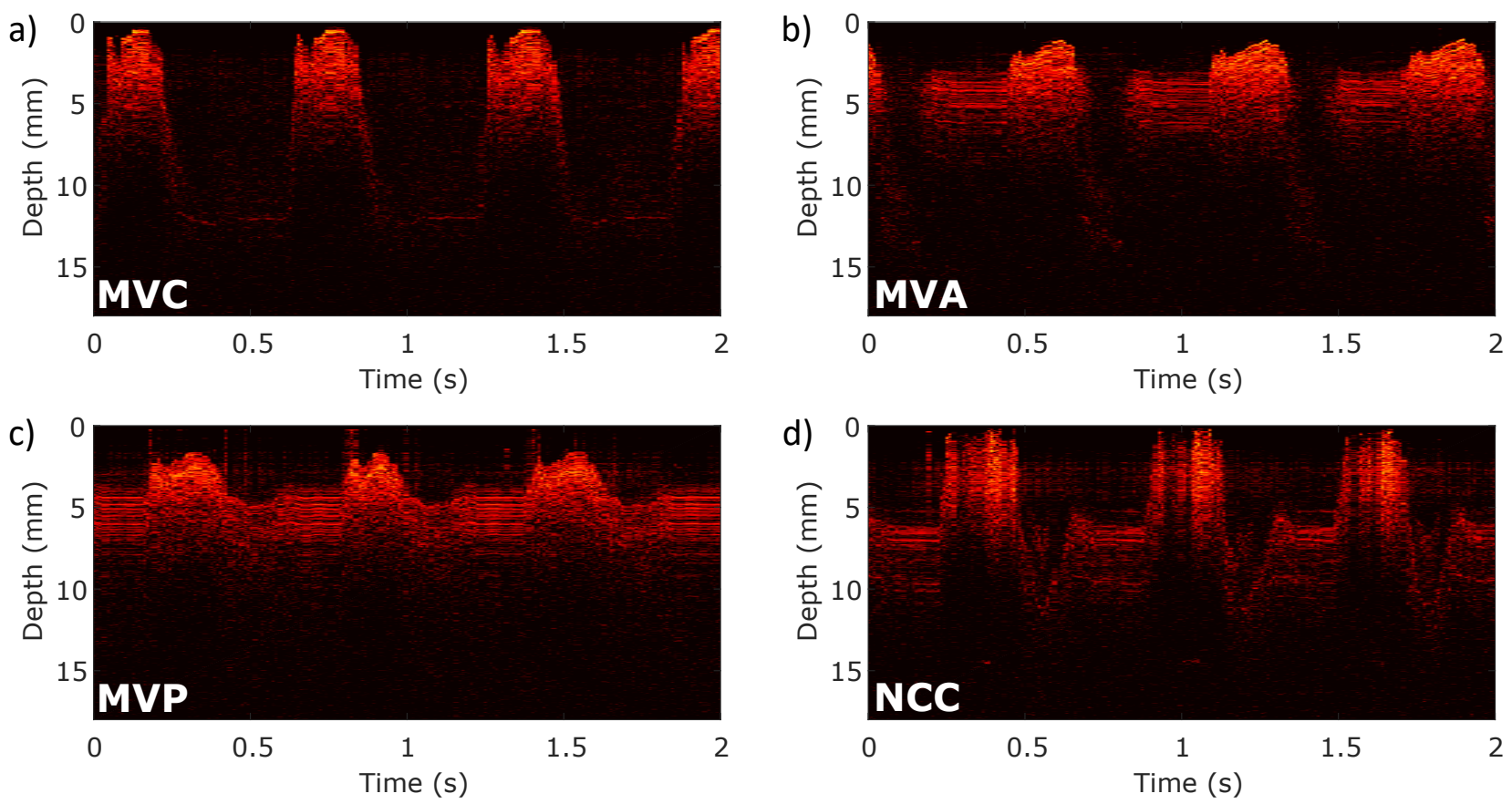

Figure 3. Snapshots of acquired all-optical ultrasound M-mode images, on the mitral valve: a) central (MVC), b) anterior (MVA), c) posterior (MVP), and on the aortic valve (NCC).

physiological conditions could be simulated with the phantom by adjusting the valves.

The device used in these experiments was specifically designed for use with the heart valve phantom; as such, a rigid hypotube was used to house the device. For future applications, this housing could be easily adapted to suite the imaging scenario. The outer diameter could be greatly reduced, since the OpUS device had an outer diameter $<1 \mathrm{~mm}$. Further, a flexible housing such as a catheter could be used to navigate more tortuous pathways. However, the flexibility is currently limited by the $600 \mathrm{\mu m}$ core diameter optical fibre which has a minimum bend radius of $24 \mathrm{~mm}$.

The OpUS images acquired were promising in terms of imaging depth $(>15 \mathrm{~mm})$, constrast, and resolution. Images were acquired at 4 locations: MVC, MVA, MVP, and NCC. For all the acquired images the valve was well visualised throughout the cardiac cycle. Crucially, the valve motion could be tracked and differences in valve motion between locations were observed, which is important for diagnosis and treatment of valve disorders. Further, the differentiation between locations on the valves is promising for navigating in a surgical scenario. This study demonstrates the potential for all-optical ultrasound imaging to be used for guidance of intracardiac interventions, including endovascular replacement and repair using all-optical ultrasound transducer integration into transcatheter devices.

\section{DISCLOSURES}

RJC, MCF \& AED own shares in Echopoint Medical Ltd. JTM \& TMP are co-owners of Archetype Biomedical Inc.

\section{ACKNOWLEDGEMENTS}

This work was supported by the Royal Academy of Engineering under the Research Fellowship Scheme $(\mathrm{RF} \backslash 201819 \backslash 18 \backslash 125)$ and the Wellcome/EPSRC Centre for Interventional and Surgical Sciences (WEISS) $(203145 / Z / 16 / Z)$ 


\section{REFERENCES}

[1] Prada, G., Vieillard-Baron, A., Martin, A. K., Hernandez, A., Mookadam, F., Ramakrishna, H., and DiazGomez, J. L., "Echocardiographic Applications of M-Mode Ultrasonography in Anesthesiology and Critical Care," Journal of Cardiothoracic and Vascular Anesthesia 33, 1559-1583 (jun 2019).

[2] Cox, B. T. and Beard, P. C., "Fast calculation of pulsed photoacoustic fields in fluids using k -space methods," The Journal of the Acoustical Society of America 117, 3616-3627 (jun 2005).

[3] Noimark, S., Colchester, R. J., Poduval, R. K., Maneas, E., Alles, E. J., Zhao, T., Zhang, E. Z., Ashworth, M., Tsolaki, E., Chester, A. H., Latif, N., Bertazzo, S., David, A. L., Ourselin, S., Beard, P. C., Parkin, I. P., Papakonstantinou, I., and Desjardins, A. E., "Polydimethylsiloxane Composites for Optical Ultrasound Generation and Multimodality Imaging," Advanced Functional Materials 28, 1704919 (feb 2018).

[4] Guggenheim, J. A., Li, J., Allen, T. J., Colchester, R. J., Noimark, S., Ogunlade, O., Parkin, I. P., Papakonstantinou, I., Desjardins, A. E., Zhang, E. Z., and Beard, P. C., "Ultrasensitive plano-concave optical microresonators for ultrasound sensing," Nature Photonics 11(11), 714-719 (2017).

[5] Colchester, R. J., Zhang, E. Z., Mosse, C. A., Beard, P. C., Papakonstantinou, I., and Desjardins, A. E., "Broadband miniature optical ultrasound probe for high resolution vascular tissue imaging," Biomedical Optics Express 6(4), 1502-1511 (2015).

[6] Colchester, R. J., Little, C., Dwyer, G., Noimark, S., Alles, E. J., Zhang, E. Z., Loder, C. D., Parkin, I. P., Papakonstantinou, I., Beard, P. C., Finlay, M. C., Rakhit, R. D., and Desjardins, A. E., "All-Optical Rotational Ultrasound Imaging," Scientific Reports 9, 5576 (dec 2019).

[7] Alles, E. J., Noimark, S., Zhang, E., Beard, P. C., and Desjardins, A. E., "Pencil beam all-optical ultrasound imaging," Biomedical Optics Express 7, 3696 (sep 2016).

[8] Finlay, M. C., Mosse, C. A., Colchester, R. J., Noimark, S., Zhang, E. Z., Ourselin, S., Beard, P. C., Schilling, R. J., Parkin, I. P., Papakonstantinou, I., and Desjardins, A. E., "Through-needle all-optical ultrasound imaging in vivo: a preclinical swine study," Light: Science 83 Applications 6, e17103 (dec 2017).

[9] Zou, X., Wu, N., Tian, Y., and Wang, X., "Broadband miniature fiber optic ultrasound generator," Optics Express 22, 18119 (jul 2014).

[10] Zhou, J., Guo, X., Du, C., and Wang, X., "Ultrasound beam steering using a fiber optic ultrasound phased array," Optics Letters 44, 5390 (nov 2019).

[11] Colchester, R. J., Alles, E. J., and Desjardins, A. E., "A directional fibre optic ultrasound transmitter based on a reduced graphene oxide and polydimethylsiloxane composite," Applied Physics Letters 114, 113505 (mar 2019).

[12] Vannelli, C., Moore, J., McLeod, J., Ceh, D., and Peters, T., "Dynamic heart phantom with functional mitral and aortic valves," in [Medical Imaging 2015: Image-Guided Procedures, Robotic Interventions, and Modeling], Webster, R. J. and Yaniv, Z. R., eds., 9415, 941503 (mar 2015). 